\title{
ASEGURAMIENTO UNIVERSAL EN SALUD EN EL PERÚ: UNA APROXIMACIÓN A 10 AÑOS DE SU IMPLEMENTACIÓN
}

\author{
UNIVERSAL INSURANCE IN HEALTH IN PERU: AN APPROXIMATION TO 10 YEARS OF ITS IMPLEMENTATION
}

David Jumpa-Armas ${ }^{1,2, a}$

\section{RESUMEN}

La política del Aseguramiento Universal en Salud estableció que el acceso a los servicios de salud se realizara por medio de la intermediación financiera de seguros de salud, estableciendo para ello cuatro ejes de "reforma": plan de beneficios, financiamiento y pagos, focalización de subsidios, prestación de servicios y regulación.

La política del Aseguramiento Universal en Salud se basó en la teoría de los cuasimercados donde la intención del Estado es evitar ser el proveedor de recursos y el proveedor de servicios al mismo tiempo; en lugar de ello, busca convertirse en el proveedor primario de fondos para una variedad de proveedores del sector privado, público y no lucrativos, todos operando en competencia unos contra otro.

A 10 años de su implementación en nuestro país se analizan los avances de implementación en los ejes de reforma que planteó la política del Aseguramiento Universal en Salud.

Palabras clave: Aseguramiento Universal en Salud; Políticas Públicas en Salud; Reforma de la atención de salud. (fuente: DeCS BIREME)

\begin{abstract}
The policy of the Universal Health Insurance established that access to health services will be carried out through the financial intermediation of health insurance, establishing for this four axes of "reform": plan of benefits, financing and payments, targeting of subsidies, service provision and regulation.

The policy of the Universal Health Insurance was based on the theory of quasi-markets where the intention of the State is to avoid being the provider of resources and the service provider at the same time; instead, it seeks to become the primary provider of funds to a variety of private, public and nonprofit providers, all operating in competition against each other.

Ten years after its implementation in our country, the progress made in the implementation of the reform axis proposed by the Universal Health Insurance policy is analyzed.
\end{abstract}

Key words: Universal Health Insurance; Public Policies in Health; Health Care Reform. (source: MeSH NLM)

\section{INTRODUCCIÓN}

La sociedad peruana ha experimentado un políticas de salud consiste en marcar la orientación de sostenido cambio demográfico en los últimos 50 largo plazo al resto de elementos del sistema de salud, años $^{1,2}$ que, junto a las condiciones de vida, factores para generar resultados intermedios en materia de de riesgo medio ambiental y del estilo de vida, acceso a servicios de salud de calidad con criterios de vienen determinando los actuales y futuros retos a eficiencia y sostenibilidad, todo ello con la finalidad la situación de salud de nuestra población (Figura 1). de mejorar el estado de salud de la población con La respuesta social y organizada a estos retos dan menos mortalidad, morbilidad y discapacidad, forma al sistema de salud del país, donde el rol de las generando así mayor bienestar social (Figura 2). 
La orientación de las políticas de salud en nuestro país no ha estado lejos del espectro de políticas económicas y sociales que, en las últimas décadas, delinearon el modelo de Estado que tenemos. Las tendencias han determinado que las garantías sociales públicas, que redistribuyen los riesgos, se debilitaran y restringieran a grupos concretos (los que fracasaron en el objetivo de asegurarse su propio bienestar) que apueste al mercado como base de la estructura de producción de bienestar y, por omisión se perpetúe las asimetrías de género al interior de las familias cuando a estas se les impone una carga en la provisión de bienestar ${ }^{3}$.

\section{La política del Aseguramiento Universal en Salud}

En tal contexto, sustentados en el enfoque

La política del AUS estableció que el acceso a los servicios de salud se realizara por medio de la intermediación financiera de seguros de salud, estableciendo para ello cuatro ejes de "reforma": plan de beneficios, financiamiento y pagos, focalización de subsidios, prestación de servicios y regulación ${ }^{5}$.

A continuación, se hará una aproximación a los resultados de la implantación de la política del AUS por medio del análisis de sus ejes de reforma.

\section{Plan de beneficios}

El plan de beneficios de salud es el listado de condiciones asegurables, intervenciones, prestaciones y garantías explícitas que ofrece un seguro de salud a sus afiliados ${ }^{6}$. El plan mínimo se denomina PEAS, fue aprobado el año 2009 y cubre procedimientos asociados a 140 condiciones asegurables y 34 garantías explícitas de oportunidad y calidad7. A pesar de que la Ley AUS establecía una evaluación bienal del PEAS para su reformulación, a diez años de su aprobación no existe información pública sobre las evaluaciones realizadas.

En efecto, el Plan de Implementación del AUS establecía que para el año 2014 el PEAS debía incluir al menos 185 condiciones asegurables y 70 garantías explícitas, nada de ello es realidad a la fecha ${ }^{8}$.

Si bien el Seguro Integral de Salud (SIS) amplió el paquete de beneficios para sus asegurados, no existe claridad sobre la capacidad de prestación de los servicios, la población efectivamente atendida o los costos de provisión de los paquetes ${ }^{9}$. Finalmente, en el caso del mercado privado de seguros de salud, la falta de actualización del PEAS constituye una limitación a la progresividad en la cobertura de prestaciones para los ciudadanos que cotizan en los planes de beneficios de las aseguradas privadas que toman como referencia al PEAS.

\section{Financiamiento y mecanismos de pago}

La Ley N²9761 (2011) estableció que el presupuesto público para el financiamiento del régimen subsidiado del SIS, tome como referencia el valor de la prima anual del PEAS y el número de afiliados ${ }^{10}$. No obstante, a la fecha, la Ley no ha sido reglamentada por el Ministerio de Salud y el financiamiento para los asegurados del SIS se realiza predominantemente por el mecanismo del presupuesto histórico.

La falta de sinceramiento de los recursos necesarios para el financiamiento de los asegurados del SIS explica en gran medida la posición relegada que aún mantiene nuestro país en materia de gasto público destinado a la salud (3,3\% del PBI para el año 2016) ${ }^{11}$, siendo que una condición necesaria para reducir las inequidades y aumentar la protección financiera pasa por sostener un gasto público destinado a la salud no menor del 6\% del $\mathrm{PBI}^{12}$.

De similar forma, en los últimos 10 años, el gasto de bolsillo (pago al recibir el servicio en forma de medicamentos, copagos o deducibles) se ha mantenido por encima del $20 \%$ del gasto total en salud hasta el año $2016^{11}$. Asimismo, el gasto catastrófico en salud (gastos en salud que exceden el $40 \%$ de la capacidad de pago del hogar) no se ha reducido significativamente en el periodo 2006 - 2016, pasó de un $5 \%$ a $4 \%$ de hogares afectados ${ }^{13}$.

Por otro lado, los mecanismos de pago para la compra de servicios de salud, que deben permitir alinear incentivos a los prestadores hacia el logro de resultados de salud, se han mostrado inefectivos y han contribuido a burocratizar los sistemas administrativos de los prestadores. La brecha entre el diseño y la implementación delmecanismo decompra de servicios de salud por medio del "Presupuesto por Resultados" no ha permitido superar el pago por insumo a un verdadero pago por resultados. En el caso del primer nivel de atención, los sistemas denominados "cápita" del SIS difieren del sistema básico de los mecanismos capitados internacionalmente, reconocidos por privilegiar sus cálculos con base en la producción histórica; finalmente, en el caso del pago a hospitales, 
el SIS realiza la compra en base a la facturación de servicios por medio de tarifarios que varían de hospital a hospital; también, reconoce los costos adicionales en los que puedan haber incurrido los hospitales; no obstante ello, son frecuentes las demoras administrativas, derivadas de las transferencias y de la gestión administrativa, al interior de los prestadores que no permiten disponer de los recursos de forma efectiva desde el primer mes de cada año?.

\section{Focalización y subsidios}

Los esfuerzos de identificación de la población en condición de pobreza utilizaron mecanismos de focalización geográfica, focalización individual, focalización por criterio de población vulnerable; sin embargo, ello contrasta con el hecho de que al menos un tercio de la población en pobreza se mantiene en tal situación (pobreza crónica) y los otros dos tercios registran salidas y entradas con relación a la línea de pobreza ${ }^{14}$. El esfuerzo focalizador contrasta con la menor atención que ha recibido la importante subcobertura del SIS, según Petrera, en base a datos del año 2014, existe una subcobertura del SIS del $68,6 \%$ para el primer quintil y del $60,4 \%$ para el segundo quintil de mayor pobreza en el ámbito de Lima Metropolitana ${ }^{15}$.

\section{Prestación de servicios}

La expansión de la oferta de establecimientos de salud en los departamentos piloto de la política del Aseguramiento Universal en Salud en Ayacucho, Apurímac, Huancavelica y Lima Metropolitana durante el periodo 2009-2018, en el subsistema del MINSA/gobiernos regionales (GR) fue de 15 (4\%), 112 (40\%), 91 (28\%) y 33 (9\%) establecimientos de salud, respectivamente. En el subsistema de ESSALUD fue de $1(9 \%), 3(60 \%), 0(0 \%)$ y $17(63 \%)$ establecimientos de salud en cada departamento.

En el subsistema del sector privado, la expansión de la oferta fue mayor con 24 (800\%), 81 (506\%), $22(76 \%)$ y 5393 (376\%) establecimientos de salud, respectivamente (Tabla 1 ).

No obstante, la población del SIS que no logró acceder a servicios de salud, a pesar de necesitar de ellos, se incrementó de $37,3 \%$ a $49,4 \%$ en el ámbito urbano y de $36,6 \%$ a 49,9\% en el ámbito rural durante el periodo 2004-2014 ${ }^{15}$; es decir, la ampliación de la oferta de establecimiento de salud del MINSA/GR ha resultado insuficiente a la demanda de servicios por parte de los afiliados al SIS. Finalmente, los esfuerzos destinados al "intercambio prestacional" (atenciones de afiliados al SIS en ESSALUD) han sido poco eficaces llegando sólo a un $1,3 \%$ del total de atenciones del SIS 9 .

\section{Regulación y Fiscalización}

La capacidad regulatoria del Ministerio de Salud en materia de aseguramiento ha sido trasladada a la Superintendencia Nacional de Salud (SUSALUD), la misma que ejerce la función de fiscalización del AUS ${ }^{16}$. Para abril del 2019, según el registro de sanciones de SUSALUD, sehan interpuesto un total 74 sanciones desde la entrada en vigencia del Reglamento de Infracciones y Sanciones en el año 2014. En el caso de las Instituciones Administradoras de Fondos de Aseguramiento en Salud (IAFAS) son 12 sanciones por un monto total de 142 UIT y en el caso de las Instituciones Prestadoras de Servicios de Salud (IPRESS) son 62 sanciones por un monto total 1558 UIT $^{17}$.

\section{Reflexiones finales}

La política del AUS priorizó en su diseño la dimensión financiera del sistema de salud, centrándose en aspectos de eficiencia asignativa y equidad en términos de protección financiera, para ello se basó en la teoría de los cuasimercados donde la intención del Estado es evitar ser el proveedor de recursos y servicios al mismo tiempo; en lugar de ello, busca convertirse en el proveedor primario de fondos para una variedad de proveedores del sector privado, público y no lucrativo, todos operando en competencia unos contra otro ${ }^{18}$. Aun, cuando resulte necesaria la externalización de servicios, resulta pertinente explicitar que no es lo mismo externalizar desde convicciones públicas que desde convicciones privadas ${ }^{3}$. En el caso peruano, durante la implementación de la política del AUS se puede apreciar una externalización de servicios de forma reactiva, poco planificada y con deficientes mecanismos de control (boticas privadas "FARMASIS", atenciones de emergencia en clínicas privadas, el "negociazo" del caso Moreno). En suma, se externalizó no buscando más eficacia y eficiencia, sino que se externalizó para evitar contratar directamente más personal.

Finalmente, es necesario mitigar los potenciales problemas de una política con deficiencias de valores públicos como el AUS; por un lado, la posibilidad de que se intensifiquen los problemas de "capturas" del regulador por el regulado, expresado en que altos funcionarios públicos que controlan el sector prosigan de forma natural sus carreras en agentes del sector privado que antes han regulado, y por otro, el riesgo del mayor control de la agenda pública por parte de un sector privado, que optó por la concentración e integración vertical conformando grandes oligopolios en contraste a la fragmentación y debilitamiento del sector público. 
Es necesaria una amplia reflexión sobre el diseño e implementación de la política del AUS en nuestro país, antes de seguir profundizando sus ejes de reforma. El autor considera que esta política no encontró el nudo decisivo para desarrollar una reforma en el sector salud.

Los interesados en las políticas de salud tienen la tarea de seguir las experiencias exitosas como es el caso de la "reforma de la salud mental". Esta política estableció una reconfiguración de la dimensión programática de la salud mental, estableció como finalidad el logro de resultados sanitarios privilegiando la efectividad, garantizó a nivel legislativo los derechos de las personas con problemas de salud mental, logró articular la eficiencia asignativa mediante el mecanismo de presupuestos por resultados y viene expandiendo la oferta pública de servicios de salud mental mediante un modelo de atención comunitaria.

La lección está dada, cambiar la vieja manera de producir salud para garantizar su acceso universal es posible y es más necesario que nunca.

\section{DALYs 1990}

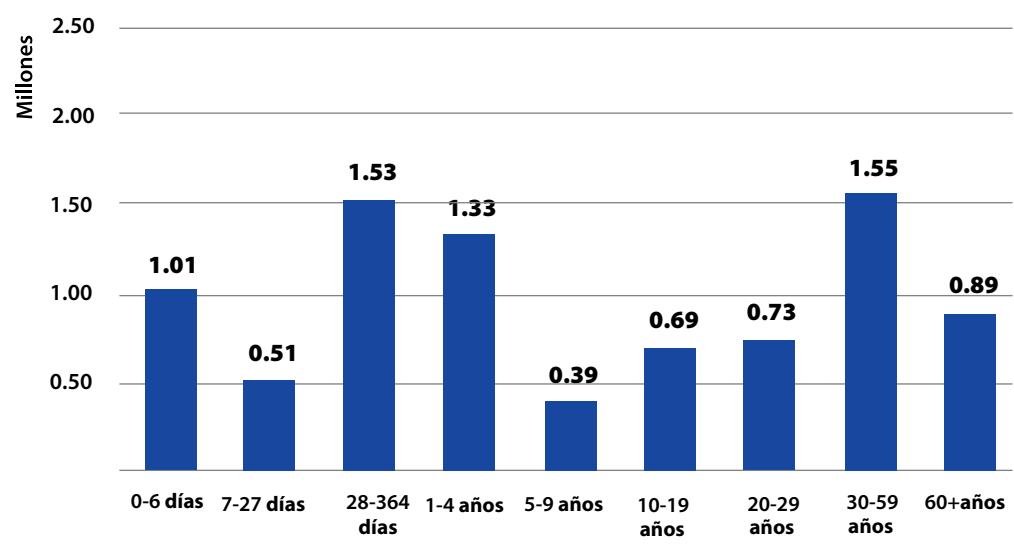

DALYs 2000

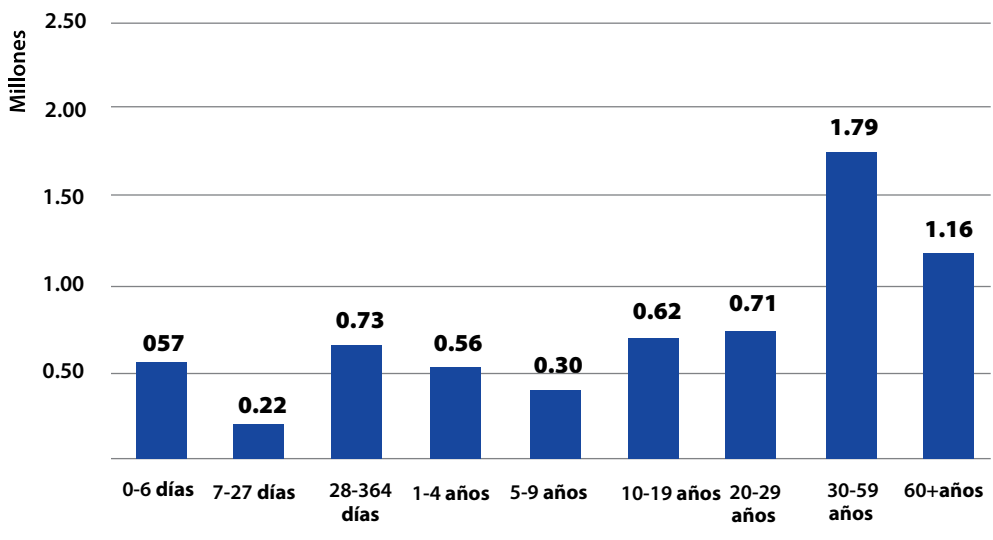

DALYs 2016

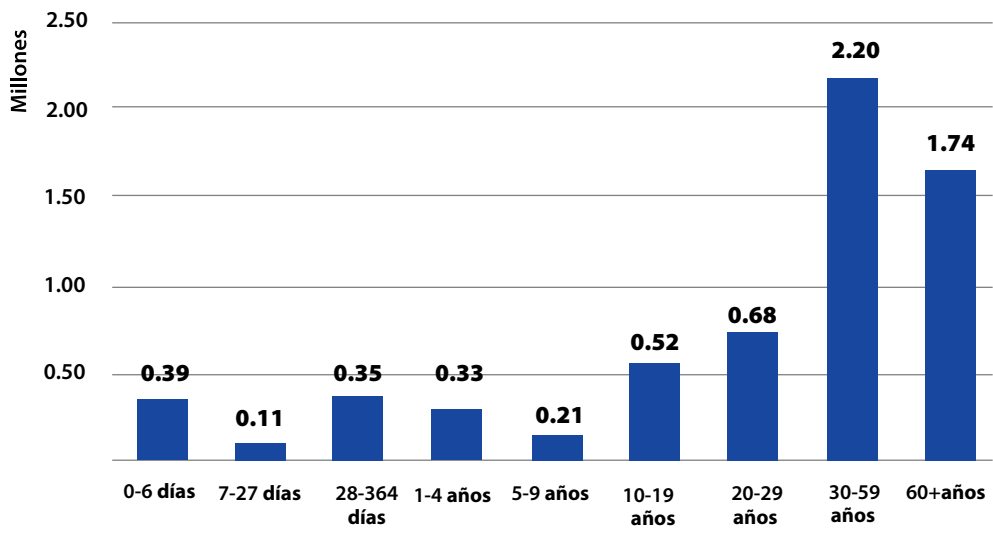

Fuente: Elaboración propia con datos del Global Burden of Disease Study 2016.

Figura1. Evolución de la carga de enfermedad en el Perú, 1990-2016. 


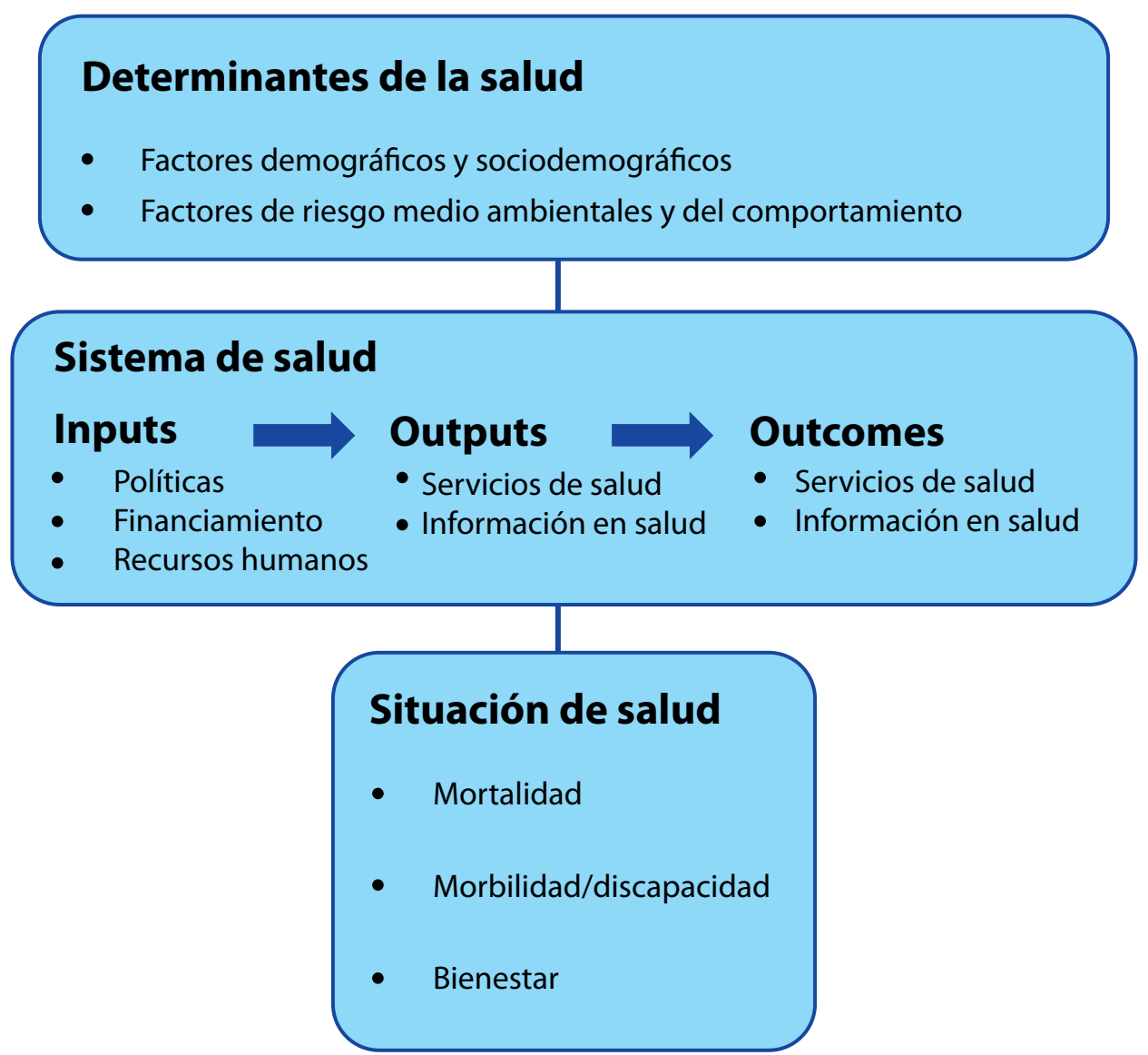

Fuente: Adaptado a partir de la Organización Mundial de la Salud (2008).

Figura 2. Dominios para el análisis del sistema de salud.

Tabla 1. Establecimientos de salud según subsistema en departamentos piloto del AUS, 2009-2018.

\begin{tabular}{|c|c|c|c|c|c|c|c|c|c|c|c|c|c|}
\hline Subsistema & Departamento & $\begin{array}{c}\text { Antes } \\
2009\end{array}$ & 2009 & 2010 & 2011 & 2012 & 2013 & 2014 & 2015 & 2016 & 2017 & 2018 & $\begin{array}{l}\text { Incremento } \\
\text { en periodo }\end{array}$ \\
\hline \multirow{4}{*}{$\begin{array}{l}\text { MINSA / } \\
\text { GOBIERNO } \\
\text { REGIONAL }\end{array}$} & Ayacucho & 414 & 420 & 424 & 426 & 426 & 427 & 427 & 427 & 428 & 428 & 429 & $15(4 \%)$ \\
\hline & Apurímac & 282 & 304 & 341 & 369 & 376 & 380 & 386 & 391 & 392 & 392 & 394 & $112(40 \%)$ \\
\hline & Huancavelica & 324 & 348 & 390 & 401 & 405 & 408 & 413 & 414 & 414 & 414 & 415 & $91(28 \%)$ \\
\hline & $\begin{array}{l}\text { Lima } \\
\text { Metropolitana }\end{array}$ & 376 & 381 & 384 & 388 & 395 & 398 & 398 & 399 & 402 & 404 & 409 & $33(9 \%)$ \\
\hline \multirow{4}{*}{ ESSALUL } & Ayacucho & 11 & 12 & 12 & 12 & 12 & 12 & 12 & 12 & 12 & 12 & 12 & $1(9 \%)$ \\
\hline & Apurímac & 5 & 6 & 6 & 7 & 7 & 7 & 7 & 8 & 8 & 8 & 8 & $3(60 \%)$ \\
\hline & Huancavelica & 8 & 8 & 8 & 8 & 8 & 8 & 8 & 8 & 8 & 8 & 8 & $0(0 \%)$ \\
\hline & $\begin{array}{l}\text { Lima } \\
\text { Metropolitana }\end{array}$ & 27 & 35 & 38 & 42 & 42 & 43 & 44 & 44 & 44 & 44 & 44 & $17(63 \%)$ \\
\hline \multirow{4}{*}{ PRIVADO } & Ayacucho & 3 & 4 & 4 & 7 & 10 & 15 & 16 & 17 & 23 & 23 & 27 & 24 (800\%) \\
\hline & Apurímac & 16 & 22 & 23 & 30 & 56 & 68 & 76 & 85 & 90 & 96 & 97 & 81 (506\%) \\
\hline & Huancavelica & 29 & 35 & 36 & 38 & 39 & 42 & 44 & 47 & 47 & 49 & 51 & 22 (76\%) \\
\hline & $\begin{array}{l}\text { Lima } \\
\text { Metropolitana }\end{array}$ & 1436 & 1951 & 2446 & 2977 & 3783 & 4472 & 5042 & 5700 & 6101 & 6534 & 6829 & $5393(376 \%)$ \\
\hline
\end{tabular}

Fuente: Registro Nacional de Instituciones Prestadores de Servicios de Salud (RENIPRESS). 
Contribuciones de autoría: El autor participó en la concepción y diseño del trabajo; recolección / obtención de datos; contribución estadística; análisis e interpretación de datos; revisión crítica del manuscrito; redacción del manuscrito y aprobación de su versión final.

Financiamiento: Autofinanciado.

Conflicto de interés: El autor declara no tener conflicto de interés en la publicación de este artículo.

Recibido: 02 de diciembre 2018

Aprobado: 22 de mayo 2019
Correspondencia: David Jumpa Armas.

Dirección: Jirón Domingo Cueto 109, Jesús María 15072, Lima-Perú.

Teléfono: 999070905

Correo:david.jumpa@upch.pe

\section{REFERENCIAS BIBLIOGRÁFICAS}

1. Perú. Instituto Nacional de Estadística e Informática. Perú: Perfil Sociodemográfico. Informe Nacional. Censos Nacioanales 2017. [Internet]. 2018. Available from: https://www.inei.gob.pe/media/ MenuRecursivo/publicaciones_digitales/Est/Lib1539/index.html

2. Institute for Health Metrics and Evaluation. Global Health Data Exchange | GHDx [Internet]. [cited 2019 May 1]. Available from: http:// ghdx.healthdata.org/

3. Ramió C. Los problemas de la implantación de la nueva gestión pública en las administraciones públicas latinas: modelo de Estado y cultura institucional [Internet]. Revista del CLAD Reforma y Democracia. 2001 [cited 2019 May 1]. Available from: http://old.clad. org/portal/publicaciones-del-clad/revista-clad-reforma-democracia/ articulos/021-octubre-2001/los-problemas-de-la-implantacion-dela-nueva-gestion-publica-en-las-administraciones-publicas-latinasmodelo-de-estado-y-cultura-institucional

4. Mendoza I. El acuerdo de partidos políticos en salud. Sistematización de experiencias. [Internet]. 2005. Available from: http://bvs.minsa.gob. pe/local/minsa/1040_GRAL1198.pdf

5. Arce M. Implementación Del Aseguramiento Universal En Salud. Rev Peru Med Exp Salud Publica. 2009;26(2):218-21.

6. Perú. Poder Ejecutivo. Decreto Supremo N 020-2014-SA. Texto Único Ordenado de la Ley $\mathrm{N}^{\circ}$ 293444, Ley Marco de Aseguramiento Universa en Salud. 2014; Available from: https://cdn.www.gob.pe/uploads/ document/file/200456/197192_Decreto_Supremo_N_C2_B0020 2014-SA.PDF20180926-32492-1ikk432.PDF

7. Poder Ejecutivo. Ministerio de Salud. Decreto Supremo $N^{\circ} 016$ 2009. Aprueban Plan Esencial de Aseguramiento en Salud [Internet] 2009. Available from: https://cdn.www.gob.pe/uploads/document/ file/274778/245991_DS016-2009Ori.pdf20190110-18386-57m3v2.pdf

8. Perú. Ministerio de Salud. Informe de Implementación de Aseguramiento Universal en Salud [Internet]. 2010. Available from: http://www.minsa.gob.pe/portada/aseguramiento/Archivos/Informe Primer_Anio_AUS.pdf

9. Vermeersch CMJ. Financiamiento de la salud en el Perú : Análisis de la situación actual y desafíos de política al 2021 [Internet]. 2016 [cited 2019 May 1]. p. 1-1. Available from: http://documentos.bancomundial.
org/curated/es/441041481748303633/Financiamiento-de-la-salud-enel-Perú-Análisis-de-la-situación-actual-y-desafíos-de-política-al-2021

10. Congreso de la República. Ley de Financiamiento Público de los Regímenes Subsidiado y Semicontributivo del Aseguramiento Universal en Salud [Internet]. 2011. Available from: https://cdn.www.gob.pe/ uploads/document/file/272217/243325_Ley29761.pdf2019011018386-11on16u.pdf

11. Organización Mundial de la Salud. Global Health Expenditure Database [Internet]. [cited 2019 May 1]. Available from: http://apps. who.int/nha/database/Home/Index/en/

12. Organización Panamericana de la Salud. Estrategia para el acceso universal a la salud y la cobertura universal de salud [Internet]. [cited 2019 May 1]. Available from: http://iris.paho. org/xmlui/bitstream/handle/123456789/28276/CD53-5-s. pdf;jsessionid=361975679E8BB2EEA58CE1800961B3EA? sequence $=4$

13. Proaño Falconi $D$, Bernabé E. Determinants of catastrophic healthcare expenditure in Peru. Int J Heal Econ Manag [Internet]. 2018;18(4):42536. Available from: https://doi.org/10.1007/s10754-018-9245-0

14. Chacaltana J. ¿Se puede prevenir la pobreza? Informe Final 2006;1-80. Available from: http://www.cies.org.pe/sites/default/files/ investigaciones/pobrezaciesfinal.pdf

15. Petrera Pavone M. El complejo proceso de la inclusión : reforma y seguro integral de salud [Internet]. [cited 2019 May 1]. 141 p Available from: http://catalogo.iep.org.pe/cgi-bin/koha/opac-detail. pl?biblionumber $=43188$

16. Poder Ejecutivo. Decreto Legislativo 1158. Decreto Legislativo que dispone medidas al fortalecimiento y cambio de denominación de la Superintendencia Nacional de Aseguramiento en Salud [Internet]. 2013. Available from: https://cdn.www.gob.pe/uploads/document/ file/200293/197022_DL1158.pdf20180926-32492-le2jzq.pdf

17. Superintendencia Nacional de Salud. Registros de Sanciones SUSALUD [Internet]. [cited 2019 May 1]. Available from: http://portal. susalud.gob.pe/registros-de-sanciones/

18. Le Grand J, Grand L, Julian. Quasi-markets and Social Policy. Econ J [Internet]. 1991 [cited 2019 May 1];101(408):1256-67. Available from: https://econpapers.repec.org/article/ecjeconjl/v_3a101_3ay_3a1991_ 3ai_3a408_3ap_3a1256-67.htm 